\title{
Sentidos sobre o hábito de fumar para mulheres participantes de grupo de tabagistas
}

\author{
Neusa da Silva Eckerdt ${ }^{1}$ \\ Clarissa Mendonça Corradi-Webster²
}

O objetivo deste estudo foi conhecer sentidos produzidos sobre o tabagismo por mulheres participantes de um grupo para tabagistas em Florianópolis, SC, Brasil. O grupo foi composto por sete participantes. Utilizou-se a entrevista estruturada, com cinco questões abertas. $\mathrm{O}$ referencial teórico foi construcionismo social. Como conclusão, encontrou-se dois eixos temáticos: 1) cigarro no ciclo vital da mulher 2 ) expectativas em relação ao tratamento. Os repertórios utilizados para descrever o início do consumo na adolescência incluíram glamour, charme, independência e aceitação pelos pares, enquanto aqueles utilizados para descrever a manutenção do consumo na idade adulta incluíram ansiedade, depressão e recurso para lidar com o estresse diário. Quanto às expectativas em relação ao tratamento, acreditam que o contato com pessoas que vivenciam as mesmas dificuldades, em relação à interrupção do consumo e sonhos de uma vida saudável, seria motivador. 0 tratamento oferecido para tabagistas deve explorar estratégias para que as mulheres alcancem o que desejam com o consumo de cigarro.

Descritores: Tabagismo; Mulheres; Terapêutica.

\footnotetext{
${ }^{1}$ Enfermeira; Mestranda em Saúde Publica, Universidade Federal de Santa Catarina, SC, Brasil. E-mail: eckerdtt@yahoo.com.br. 2 Psicóloga; Doutor em Psicologia, Pesquisadora, Escola de Enfermagem de Ribeirão Preto, Universidade de São Paulo, Centro Colaborador de la OMS para el Desarrollo de la Investigación en Enfermería, SP, Brasil. E-mail: clarissac@usp.br.
}

Correspondencia:

Clarissa Mendonça Corradi-Webster

Universidade de São Paulo. Escola de Enfermagem de Ribeirão Preto

Av. Bandeirantes, 3900

Bairro Monte Alegre

CEP: 14040-902 Ribeirão Preto, SP, Brasi

E-mail: clarissac@usp.br 


\section{Significados acerca del fumar para las mujeres participantes en un grupo de fumadores}

El objetivo de este estudio fue conocer el significado, sobre el fumar, declarado por mujeres que participaban en un grupo de fumadores en Florianópolis, estado de Santa Catarina, Brasil. Participaron siete fumadores. Se utilizó entrevistas estructuradas con cinco preguntas abiertas. El marco teórico fue el construccionismo social. Dos temas fueran detectados: 1) Los cigarrillos en el ciclo de vida de la mujer 2) Expectativas en relación al tratamiento. Los repertorios utilizados para describir el inicio del consumo, en la adolescencia, incluyen: glamour, encanto, independencia y el ser aceptado entre iguales; y los utilizados para describir el mantenimiento del consumo en la edad adulta incluyen: ansiedad, depresión y el uso como recurso para lidiar con el estrés diario. En cuanto a las expectativas para el tratamiento, creen que ayudaría el contacto con personas que experimentan las mismas dificultades en relación con la interrupción del consumo; también que los sueños de una vida saludable sería motivador. El tratamiento ofrecido a fumadores debería explorar estrategias para las mujeres que quieren abandonar el consumo de cigarrillos.

Descriptores: Tabaquismo; Mujeres; Terapêutica.

\section{Meanings about Smoking for Women Participant in a Group for Smokers}

The aim of this study was to understand the meanings produced about smoking by women participating in a group for smokers in Florianopolis, SC, Brazil. Seven participants took part in structured interviews with five open questions. The theoretical framework was social constructionism with two themes: 1 ) Cigarettes in the women's life cycle 2) Expectations regarding treatment. The repertories used to describe the beginning of consumption in adolescence included glamour, charm, independence and peer acceptance and those used to describe the maintenance of consumption in adulthood included anxiety, depression and coping with daily stress. Regarding expectations for treatment they believed that contact with people who experience the same difficulties in relation to the cessation of consumption and the dream of a healthy life would be motivating. The treatment offered to smokers should explore strategies for women to achieve what they want regarding the consumption of cigarettes.

Descriptors: Smoking; Women; Therapeutics.

\section{Introdução}

O uso do tabaco e suas consequências são vistos como importantes problemas de saúde coletiva, aparecendo como uma das principais causas de morte. Devido a isso, ações de prevenção e controle encontramse entre as prioridades para a Organização Mundial de Saúde (OMS) e para o Ministério da Saúde (MS), do Brasil. Nos dias de hoje, aproximadamente $47 \%$ da população masculina e $12 \%$ da população feminina no mundo fumam(1).
A literatura aponta que, em relação ao gênero, há diferença no consumo de tabaco entre países desenvolvidos e em desenvolvimento. Em países em desenvolvimento, a população masculina fumante é maioria, perfazendo $48 \%$ da população e, em países desenvolvidos, em torno de $24 \%$ da população feminina é fumante. Através de alguns estudos realizados, podese afirmar que o número de mulheres que fumam está aumentando em proporção maior do que o número 
de homens, principalmente nas faixas etárias mais jovens $^{(1)}$.

Pela maior participação das mulheres no mercado de trabalho e com conquistas relacionadas ao poder de decisão e independência financeira, a indústria do tabaco as tem colocado cada vez mais na mira de suas campanhas, principalmente tentando convencê-las de que o cigarro reflete uma imagem de emancipação e independência(2). As mulheres, atualmente, têm adotado posição mais dominante socialmente, com poder aquisitivo significante no mercado de trabalho e, devido à sua independência financeira e expectativa de vida maior que a dos homens, têm-se tornado os principais alvos das propagandas de cigarro. Segundo revisão realizada pela $\mathrm{OMS}^{(3)}$, as mulheres, frequentemente, fumam em resposta a situações negativas de vida, em situações de estresse, assim como também o usam para moderar emoções, para suprir, particularmente, a raiva. O fator manutenção do peso corporal também aparece como um dos motivos para não parar de fumar. A relação da imagem corporal e o risco de ganhar peso fazem algumas mulheres usarem o tabaco para gerenciá-lo, portanto, o medo de ganhar peso desmotiva-as para a possível tomada de decisão quanto a cessar o uso do tabaco(3).

Vários estudos ${ }^{(4-6)}$ apontam os fatores prejudiciais que resultam do uso do tabaco na saúde da mulher. $\mathrm{O}$ organismo feminino é muito suscetível aos efeitos nocivos do fumo. Em relação à saúde reprodutiva, a mulher tabagista tem maior probabilidade de infertilidade, início precoce da menopausa e tem, ainda, o risco para desenvolver câncer do colo do útero e dismenorreia. No período gestacional, podem ocorrer problemas fetais devidos à insuficiência útero-placentária, relacionados à nicotina, resultando em risco de aborto, problemas cardiovasculares, principalmente se associados ao uso de anticoncepcionais.

O tabagismo é um agravo complexo e o seu controle requer a integração de diversos tipos de tratamento. $\mathrm{O}$ conhecimento do perfil do fumante brasileiro também possibilita a adoção de tratamentos mais eficazes e a realização de programas e campanhas de prevenção ao fumo(7-9).

No âmbito do Sistema Único de Saúde (SUS), foi instituído o tratamento do tabagismo, pela Portaria $\mathrm{n} \cdot 1035 / \mathrm{GM}$, de 31 de maio de 2004(10), regulamentada pela Portaria SAS/MS/n.442, de 13 de agosto de 2004(11). Tem como princípio norteador o tratamento para o tabagismo na oferta de psicoterapias de grupo, por meio da abordagem cognitivo-comportamental, incluindo a disponibilidade de medicamentos (antidepressivos e reposições de nicotina com adesivos e gomas de mascar), quando necessário. Realizado através da Atenção Básica, financiado pelo Piso de Atenção Básica - PAP, pode ser efetivado por qualquer equipe de saúde integrante do SUS. Tem como condição primordial a realização do credenciamento para o tratamento, devendo os profissionais realizar o treinamento específico e, regularmente, enviarem os relatórios contendo as estimativas de atendimento e o quantitativo de insumos necessários, que são os medicamentos, as gomas de mascar e adesivos com nicotina(12).

Considera-se de suma importância a avaliação sobre a percepção por parte das mulheres fumantes a respeito do uso do cigarro, compreendendo que a construção de sentidos sobre esse consumo influenciará seus hábitos. Dessa forma, o objetivo deste estudo foi conhecer os sentidos produzidos a respeito do consumo de tabaco por mulheres participantes de um grupo para tabagistas, de um Centro de Saúde do Distrito Sanitário Sul, no município de Florianópolis, SC, Brasil.

\section{Métodos}

Realizou-se pesquisa caracterizada como estudo descritivo, com abordagem qualitativa, visando descrever significados atribuídos ao consumo de tabaco. O referencial teórico utilizado foi o construcionismo social, referencial esse através do qual se estende que as pessoas estão a todo momento construindo sentido sobre suas experiências. Essas construções de sentido influenciam o modo como conduzem sua vida, e essas se dão através da linguagem, que não representa a realidade e, sim, a constrói. Dessa forma, por meio da pesquisa construcionista social, se entende a ciência como prática discursiva, sendo o conhecimento científico uma prática construída por pessoas em interação e não algo apreendido do mundo. Com isso, a pesquisa construcionista social não busca se aproximar da verdade, mas auxiliar a abertura de novas possibilidades de construção de sentido sobre determinado objeto(13-15)

Foi realizada a coleta de dados em um Centro de Saúde, no município de Florianópolis, localizado na região litorânea do Estado de Santa Catarina. O instrumento utilizado foi a entrevista estruturada, direcionada pelas questões investigativas sobre as razões para o início do uso de tabaco, os momentos em que fumaram mais e em quais fumaram menos, mudanças que gostariam que acontecessem em suas vidas, caso conseguissem parar de fumar, e sobre a expectativa em relação à participação no grupo de tabagistas. 
As participantes do estudo foram as mulheres integrantes de um grupo de tabagistas que pretendem parar de fumar. 0 grupo contava com 10 participantes ao todo, sendo 7 mulheres e 3 homens. Ao se realizar o convite, todas as mulheres presentes aceitaram participar do estudo. Dessa forma, iniciou-se a leitura do termo de consentimento livre e esclarecido, solicitando a assinatura do mesmo. Conforme o objetivo, a seleção da amostra foi intencional, participando do estudo somente as mulheres integrantes do grupo para tabagistas, sendo essas ainda fumantes, ou tendo parado de fumar desde o momento em que iniciaram sua participação no grupo.

Algumas falas significativas foram acrescentadas na análise para oferecer uma visão representativa aos eixos temáticos estudados. A fim de preservar o anonimato, as mulheres entrevistadas e suas falas, foram aqui numeradas de 1 a 7 .

A análise dos dados foi realizada de acordo com os seguintes passos: 1 ) imersão dos dois autores no material coletado, 2) confronto entre os sentidos produzidos na leitura das entrevistas e o conhecimento prévio do campo de estudo, 3) construção dos eixos temáticos, 4) seleção de trechos da entrevista que ilustrem os eixos temáticos e 5) discussão, com base na literatura, dos conteúdos construídos.

A realização da pesquisa foi vinculada e certificada pelo Protocolo n.314/08 do Comitê de Ética em Pesquisa com Seres Humanos da Universidade Federal de Santa Catarina, assim como teve a anuência da Comissão de Acompanhamento dos Projetos de Pesquisa em Saúde na Prefeitura Municipal de Florianópolis. Foram consideradas as normas da Comissão de Ética com seres humanos, conforme a Resolução 196/96, do Conselho Nacional de Saúde.

\section{Resultados}

Serão apresentados, a seguir, os resultados deste estudo que emergiram a partir das falas analisadas. Pretende-se, assim, aproximação da compreensão do fenômeno pesquisado, bem como ampliar o olhar para o sentido dado pelas mulheres tabagistas. Depois de se realizar a análise, identificou-se dois eixos temáticos: Eixo 1 - O cigarro no ciclo vital da mulher e Eixo 2 Expectativas em relação ao tratamento.

\section{O cigarro no ciclo vital da mulher}

A seguir, será apresentado o sentido dado pelas mulheres ao ato de fumar, relacionado aos períodos da vida.

\section{Juventude: charme, glamour e independência}

As participantes relataram ter iniciado o uso de tabaco quando jovens, sendo a média da idade de início do uso 17 anos ( $d p=2,23 ; \min =14$ e máx=19). Descreveram como causa importante para o início do consumo a ideia que imaginavam estar atrelada à mulher charmosa, glamorosa e independente.

Ao atrelarem essa imagem ao consumo, optam por iniciá-lo e esse aparece então utilizado como forma de linguagem, de comunicar ao mundo a posição que desejavam assumir: mulheres bonitas e independentes. Descreveram o desejo de assumir essa posição como modo de serem aceitas pelo grupo de pares, suportando o mal-estar inicial provocado pelo consumo, a fim de obterem o desejado charme e glamour. Imagino que seja por achar bonito, ver as pessoas fumando e achar bonito. Eu mesma comecei porque achava bonito, foi no tempo do colégio, todos, colegas e amigos, fumavam e era bonito fumar na época. Fumar dava glamour, charme, era a passagem para ser aceita na turma. Eu tive dificuldade de aprender a fumar, mas para ser aceita na turma eu insisti. Às vezes me trancava no banheiro da escola e tentava aprender a fumar, me sufocava com a fumaça e tudo, mas aprendi e agora... (Participante 4).

Contribuindo para a construção da imagem positiva do consumo de tabaco na adolescência, as participantes também apontam o consumo feito por familiares. Tinha também a influência de fumantes em casa, meus pais fumavam e então para mim era normal fumar, fazia parte, não tive nenhum obstáculo para iniciar a fumar (Participante 6).

Idade adulta: ansiedade, stress e depressão

O sentido construído sobre o início do consumo aparece como algo relacionado ao mundo externo, ao desejo de ser aceito por um grupo social e de passar uma imagem de beleza. Entretanto, ao construírem um sentido para o consumo de tabaco durante a vida, pensando nos momentos em que fumaram mais, surgem descrições sobre o mundo interno, sobre sentimentos de depressão, estado de humor ansioso e estresse. Fumava mais quando estava ansiosa, quando me dava ansiedade eu acendia um em cima do outro. Às vezes fico ansiosa por besteira, às vezes com algum problema na família (Participante 1 ).

A manutenção do consumo aparece construída como associada às pressões relacionadas ao "ser adulto", como lidar com dificuldades no trabalho e com problemas na família. O sentido dado ao ato de fumar perde o glamour da adolescência e passa a ser considerado como um apoio para lidar com as dificuldades do dia a dia e com as emoções indesejadas. Fumei mais quando tinha muita 
pressão no trabalho, por ansiedade e depressão... Uso o cigarro para fugir da pressão do trabalho principalmente quando tem muitas coisas pra fazer (Participante 6).

$\mathrm{Na}$ idade adulta, os significados construídos em relação ao cigarro afastam-se desse como instrumento utilizado para provar a independência, e é construído como algo que Ihes causa dependência, sendo isso algo indesejado. Acho que é pra provar algo pra sociedade, às vezes por influência do meio, porque as amigas fumam. No meu caso, foi pela apelação da época em que comecei a fumar, as amigas fumavam, fui na onda, achava bonito, charmoso, fumava pois achava que quando quisesse parar eu parava, mas depois a gente vê que não é tão fácil assim de parar (Participante 2).

Percebe-se uma mudança de significados relacionados à aceitação social do uso de cigarros e à pressão social relacionada ao consumo. Enquanto na juventude havia uma pressão, principalmente por parte dos amigos, para fumar e com isso serem aceitas pelo grupo, na idade adulta passam a sofrer pressões da família para deixar o consumo, além de perceberem certa rejeição da sociedade a esse comportamento. Ah... minha saúde, vai acontecer uma melhora da minha saúde. Ter a casa sem cheiro de tabaco. Vou deixar meus filhos mais felizes, porque eles me cobram bastante para parar de fumar (Participante 4).

Essa mesma mudança de significado aparece na tensão relatada pela relação com o cigarro: acreditam que esse pode acalmá-las e aumentar a autoconfiança, ao mesmo tempo em que têm informações sobre os diversos problemas graves de saúde, podendo ser causados pelo cigarro. Essa tensão gera um sentimento de traição. Estava doente e sofrendo pressão para parar de fumar. Eu muitas vezes me sentia ansiosa, sozinha, solidão mesmo, uma angústia, depressão. O cigarro é como meu amigo, acabou comigo, mas não me larga (Participante 3).

Percebem então que precisam fazer algo para cuidar da sua saúde, e prevenirem possíveis agravos. Ressaltase que a média de idade das mulheres entrevistadas foi de 51,6 anos ( $d p=9,2 ; \min =41$ e $\max =69$ ), idade em que as mulheres passam por transformações fisiológicas e psicológicas, específicas dessa etapa do ciclo vital, como a menopausa, aposentadoria, luto pelo corpo jovem e mudança nos papéis sociais. Começam também a perceber-se mais vulneráveis e frágeis frente a situações de doenças.

Dessa forma, começam a sentir no corpo os efeitos indesejáveis do consumo de tabaco acumulados durante um longo período, já que, no grupo entrevistado, a média de anos de tabagismo foi de 34,6 anos ( $d p=8,87$; $\min =23$ e máx $=50$ ). Relatam também se preocuparem porque conhecem outras pessoas que tiveram prejuízos na saúde devido a esse consumo. Com isso, mostram que se percebem como mais suscetíveis para desenvolver um problema de saúde que pode ter consequências graves, desejando preveni-lo. Tenho sentido muita tosse e vi pessoas muito doentes, com pressão alta por causa do cigarro. Minha decisão também está por ver pacientes e familiares sofrendo devido ao uso de cigarros e não quero me encontrar na mesma situação (Participante 7).

Desejam, ao parar de fumar, melhorar a saúde, destacando a respiração e o condicionamento físico. Imaginam também que conseguirão, com isso, melhorar a qualidade de vida e ter autoestima mais elevada.

\section{Expectativas em relação ao tratamento}

Conforme relatado pelas entrevistadas, o início do consumo esteve relacionado a pressões externas e essa iniciação foi realizada em grupo. Para auxiliar na interrupção do hábito de fumar, essas mesmas mulheres demonstram expectativas de que, participando de um grupo para tabagistas, possam se aproveitar do contato com outras pessoas que vivenciam as mesmas dificuldades para interromper o consumo. Acho que será muito importante participar do grupo, pois quando a gente escuta que outras pessoas estão tendo as mesmas dificuldades parece que temos mais força pra lutar (Participante 3).

Ao mesmo tempo em que dividem as dificuldades, apoiam-se nos mesmos sonhos de uma vida mais saudável depois da mudança de comportamento. É muito importante a pessoa querer parar de fumar, largar o vício... mas sozinho é muito difícil... por isso quando eu venho no grupo e tenho as orientações dos profissionais de saúde parece que fica um pouco mais fácil, claro que a medicação também ajuda.... e, em casa, a família também gosta muito e isto te deixa feliz te fortalece (Participante 5).

Buscamapoio para superaressa fase, compartilhando experiências e dificuldades do tratamento. Como exposto por elas, vários são os fatores que auxiliam no sucesso do abandono do tabagismo, entre eles a própria decisão de abandono, o apoio recebido tanto de familiares como dos participantes do grupo e as estratégias apresentadas pelos profissionais, durante a realização dos encontros.

\section{Discussão}

Ao relatarem o início de consumo de tabaco, os repertórios interpretativos utilizados são: charme, glamour, independência e desejo de serem aceitas pelo grupo. Assim como observado em outro estudo(16), a mulher, ao segurar o cigarro, visualiza um sentido 
de atração para os homens, ao ancorar a imagem de mulher madura, adulta, charmosa e segura de si, no ato de fumar.

Esses repertórios condizem com as tarefas discutidas por teóricos do desenvolvimento, no que diz respeito à adolescência. O adolescente teria como importante tarefa o desenvolvimento de valores próprios, de autonomia e independência, contando com o apoio do grupo de pares para essa jornada. Dessa forma, utilizariam diferentes recursos a fim de serem aceitos pelos amigos. Conforme se pode comparar com outros estudos $^{(9,16-17)}$, o adolescente busca a aceitação de sua presença no grupo, pois, para ele, o fazer parte produz o sentido de ser aceito, de transpor a fase da adolescência para a fase adulta. Com base no estudo da $\mathrm{OMS}^{(3)}$, dados referentes à adolescência revelam que cerca de 100.000 jovens iniciam o ato de fumar a cada dia, e a idade média do início é de 15 anos. A adolescência firma-se como marco em vários estudos ${ }^{(17-19)}$, como ponto de partida da fase da experimentação e aquisição do hábito de fumar, sendo esse um dos fatores mais evidentes para a dependência do cigarro, assim como a influência de amigos e familiares.

O início do uso de tabaco aparece construído como algo que Ihes ajudou a conquistar importantes metas na juventude. Essas construções explicam também a tensão que aparece quando contam sobre o consumo atual. Os repertórios interpretativos utilizados para falar sobre o hábito de fumar, na vida adulta, incluem as estratégias para lidar com o humor deprimido, com a ansiedade, com dificuldades no trabalho e no dia a dia, dependência e problemas de saúde( ${ }^{(9)}$. São construções que, por um lado, como na adolescência, falam sobre o uso do tabaco para lidar com as tarefas da vida de adulto (exemplo: trabalho e família) e, por outro lado, dão voz às orientações de saúde recebidas, onde o cigarro é colocado como fator de risco para doenças e, seu consumo, como dependência.

A participação em grupos de apoio, como evidenciado pelas respostas das entrevistadas, é de suma importância. Constitui válvula de escape para dividir as dificuldades e compartilhar os desafios superados, assim como a abordagem utilizada pelos profissionais de saúde fortalece a decisão para parar de fumar. Podese destacar que a influência do meio e das pessoas com quem se convive pode interferir na utilização do tabaco. Pressões de grupo de amigos e colegas são modelos de comportamento que se tornam incentivadores da prática do uso do tabaco, assim como a convivência com irmãos ou pais fumantes.
Acredita-se, assim como outros estudos já publicados $^{(9,20-21)}$, que este artigo possa reforçar a importância dos grupos de apoio para o suporte de pessoas que desejam parar de fumar, destacando aqui a especificidade da importância desses grupos para o público feminino.

\section{Considerações Finais}

O tratamento para cessão do uso de tabaco deve levar em consideração os sentidos dados pelas mulheres para esse consumo e incluir estratégias que as auxiliem a encontrar o que desejam como charme, aceitação social, alívio do estresse, companhia de modo mais saudável e que não prejudique sua saúde. Salientase a importância da abrangência no atendimento e entendimento do sofrimento expressado pelas fumantes ao decidirem terminar com o hábito ou vício de fumar, assistindo as mesmas de forma integral.

Entende-se que é de suma importância avançar na proposta de atendimento em grupos educativos, levando em conta a fala dos participantes, entendendo o significado da decisão de parar de fumar. Por se tratar de um tema complexo, as novas propostas pedagógicas, ao privilegiar as vivências e experiências, em uma metodologia dialógica, abrangem maior significado e maiores possibilidades de sucesso.

$\mathrm{O}$ ato de parar de fumar, ao ser trabalhado, deve considerar as diversas manifestações de valor dado ao ato, assim como o envolvimento de conflitos únicos de cada participante e a dimensão das variáveis sociais, políticas e econômicas. Observa-se que esse fato é pouco trabalhado nos atendimentos ao fumante, o que condiz com a relevância deste estudo para que se possa alertar alguns profissionais a ampliarem o olhar sobre a questão da abordagem ao fumante.

Este estudo revelou questões referentes às propagandas de uso do tabaco como estimulantes ao ato de fumar. Atualmente, o cenário da propaganda já difere e muito do tempo em que as mulheres entrevistadas neste estudo tinham como marco do início do consumo, o que vem reforçar e apoiar todos os esforços no sentido das proibições de veiculação de propagandas nos meios de comunicações, assim como restrições aos locais permitidos aos fumantes. Vale reforçar que os objetivos já alcançados e os desafios a serem enfrentados dependem, além de programas e atitudes governamentais, também de toda e qualquer ação de prevenção aos agravos e vontade da sociedade como um todo. 


\section{Agradecimentos}

Agradecemos a Comissão Interamericana para - Controle do Abuso de Drogas/CICAD da Secretaria de Segurança Multidimensional/SSM da Organização dos Estados Americanos/OEA, a Secretaria Nacional de Políticas sobre Drogas/SENAD do Gabinete de Segurança Institucional/Brasil, a Escola de Enfermagem de Ribeirão Preto da Universidade de São Paulo e o
Centro Colaborador da Organização Mundial da Saúde para o Desenvolvimento da Pesquisa em Enfermagem, a população representada nas pesquisas, bem como as autoridades das universidades representadas pelos participantes do Programa On-Line de Especialização em Pesquisa sobre o Fenômeno das Drogas - PREINVEST, períodos 2005, 2006, 2007 e 2008.

\section{Referências}

1. Ministério da Saúde (BR). Secretaria Nacional de Atenção a Saúde. Instituto Nacional do Câncer. Tabagismo: dados e números. [internet]. [acesso em: 15 maio 2009]. Disponível em: http://www.inca.gov.br/tabagismo/frameset. asp?item $=$ dadosnum\&link $=$ mundo. $h t m$.

2. Amos A. How women are targeted by the tobacco industry .World Health Forum (WHO). 1990;11(4):416-22.

3. World Health Organization. The Tobacco Atlas. [internet]. 2002. [acesso em: 13 jun 2009]. Disponível em: http:// whqlibdoc. who.int/publications/2002/9241562099.pdf .

4. Samet JM, Yoon SY, editors. Women and the tobacco Epidemic: Challenges for the 21st Century [internet]. Canada: World Health Organization in collaboration with the Institute for Global Tobacco Control - Johns Hopkins School of Public Health; 2001. [acesso em: 13 jun 2009]. 222 p. Disponível em: http:// www.who.int/tobacco/media/en/WomenMonograph.pdf 5. Mello PRB, Pinto GR, Botelho C. Influência do tabagismo na fertilidade, gestação e lactação. J Pediatr. 2001; 77(4):257-64. 6. Horta BL, Victora CG, Barros FC, Santos IS, Menezes AMB. Tabagismo em gestantes de área urbana da região Sul do Brasil, 1982 e 1993. Rev. Saúde Pública. 1997;31(3):247-53.

7. Possato M, Parada CMGL, Tonete VLP. Representação de gestantes tabagistas sobre o uso do cigarro: estudo realizado em hospital do interior paulista. Rev Esc Enferm USP. 2007; 41(3):434-40.

8. Focchi GRA, Braun IM. Tratamento farmacológico do tabagismo. Rev. Psiquiatr. Clín. 2005; 32(5):267-75.

9. González CRV, Zago MMF. Creencias en fumadores pertenecientes a un programa de salud cardiovascular. Rev. Latino-Am. Enfermagem [internet]. 2004. [acesso em: 17 março 2010]; 12(spe): 412-9. Disponível em: http:// www.scielo.br/scielo.php?script $=$ sci_arttext\&pid=S0104$11692004000700017 \&$ Ing $=$ pt.

10. Portaria n. 1035/GM de 31 de maio de 2004 (BR). Dispõem sobre o acesso à abordagem e tratamento do tabagismo no SUS e dá outras providências. 1991. [acesso em: 04 jun 2009]. Disponível em: http://dtr2001.saude.gov.br/sas/PORTARIAS/ Port2004/GM/GM-1035.htm .
11. Portaria n. 442 de 13 de agosto de 2004 (BR). Dispõem sobre o plano para implantação da abordagem e tratamento do tabagismo no SUS e dá ouras providências. 2004. [acesso em: 04 jun 2009]. Disponível em: http://dtr2001.saude.gov.br/sas/ PORTARIAS/Port2004/PT-442.htm .

12. Ministério da Saúde (BR). Secretaria Nacional de Atenção a Saúde. Instituto Nacional do Câncer. Coordenação de Prevenção e Vigilância - Conprev. Deixando de fumar sem mistérios. Rio de Janeiro: MS/INCS; 2004. $12 \mathrm{p}$.

13. Spink MJ. Práticas discursivas e produção de sentidos no cotidiano: aproximações teóricas e metodológicas. $3^{a}$ ed. São Paulo: Cortez; 2004

14. Guanaes C. A construção da mudança em terapia de grupo. São Paulo: Vetor; 2006. 287 p.

15. Corradi-Webster CM, Carvalho AMP. Mulheres: discursos sobre o consumo de álcool e o tratamento para dependência. In: Luis MAV, Miasso AI, organizadores. Livro de Programas e Resumos do $10^{\circ}$ Encontro de Pesquisadores em Saúde Mental e Especialistas em Enfermagem Psiquiátrica. Ribeirão Preto (SP); 2008. p. AAD02.

16. Martinez ASJ, Ribeiro CRO. Em busca da igualdade: representações do ato de fumar em mulheres adolescentes. Rev. Latino-Am Enfermagem. 2008;16(spe):640-5.

17. Silva MAM, Rivera IR, Carvalho ACC, Guerra JAH, Moreira TCA. Prevalência e variáveis associadas ao hábito de fumar em crianças e adolescentes. J. Pediatr. 2006; 82(5):365-70.

18. Ministério da Saúde (BR). Vigescola Vigilância de tabagismo em escolares. Dados e fatos de 12 capitais brasileiras. Instituto Nacional do Câncer. [internet]. 2004. [acesso em: 14 jun 2009]. Disponível em: http://www.inca.gov.br/tabagismo/31maio2004/ vigescola.pdf .

19. Fraga S, Ramos E, Barros H. Uso de tabaco por estudantes adolescentes portugueses e fatores associados. Rev. Saúde Pública. 2006; 40(4):620-6.

20. Echer IC, Barreto SSM. Determinação e apoio como fatores de sucesso no abandono do tabagismo. Rev. Latino-Am. Enfermagem [internet]. 2008. [acesso em: 17 de março 2010]; 16(3): 44551. Disponível em: http://www.scielo.br/scielo.php?script=sci_ arttext\&pid $=$ S0104-11692008000300018\&lng $=$ pt.

21. Ruiz MR, Andrade D. La familia y los factores de riesgo relacionados con el consumo de alcohol y tabaco en los niños y adolescentes (Guayaquil-Ecuador). Rev. Latino-Am. Enfermagem [internet]. 2005. [acesso em: 17 maço 2010]; 13(spe):813-8 Disponível em: http://www.scielo.br/scielo.php?script=sci_ arttext\&pid=S0104-11692005000700008\&lng=pt 